FSU-HEP-980509

UH-511-902-98

BNL-HET-98/23

\title{
LHC Reach For Gauge Mediated Supersymmetry Breaking Models Via Prompt Photon Channels
}

\author{
Howard Baer ${ }^{1}$, Pedro G. Mercadante ${ }^{2}$, Frank Paige ${ }^{3}$, Xerxes Tata ${ }^{2}$ and Yili Wang ${ }^{2}$ \\ ${ }^{1}$ Department of Physics, Florida State University, Tallahassee, FL 32306, USA \\ ${ }^{2}$ Department of Physics and Astronomy, University of Hawaii, Honolulu, HI 96822, USA \\ ${ }^{3}$ Physics Department, Brookhaven National Laboratory, Upton, NY 11973, USA
}

(February 17, 2018)

\begin{abstract}
We evaluate the supersymmetry reach of the Large Hadron Collider within the gauge-mediated low energy supersymmetry breaking framework, assuming that a neutralino is the second lightest sparticle and that it decays promptly into a gravitino which escapes detection. We find that the maximum reach is obtained via a search for inclusive $\gamma \gamma+E_{T}$ events coming dominantly from chargino and neutralino production. Assuming an integrated luminosity of $10 \mathrm{fb}^{-1}$, we find that LHC experiments will be able to probe values of the model parameter $\Lambda \lesssim 400 \mathrm{TeV}$, corresponding to $m_{\tilde{g}} \leq 2.8 \mathrm{TeV}$. A measure of the model parameter $\Lambda$ may be possible from the photon $p_{T}$ spectrum.
\end{abstract}


Most phenomenological analyses [1] of supersymmetry (SUSY) have been carried out within the framework of the mSUGRA model [2], where gravitational interactions induce effects of SUSY breaking (assumed to occur in a "hidden sector") into the "observable sector" of ordinary particles and their superpartners. In this case, the particle-sparticle mass gap in the observable sector is $M_{\text {obs }} \sim\langle F\rangle / M_{\text {Planck }}$, where $\sqrt{\langle F\rangle}$ is the scale at which SUSY is broken. To ensure that SUSY can stabilize the elementary scalar electroweak symmetry breaking sector, we require $M_{\text {obs }} \sim 250 \mathrm{GeV}$, and so choose $\langle F\rangle \sim\left(10^{11} \mathrm{GeV}\right)^{2}$. The gravitino, which acquires a mass $\sim M_{o b s}$, then decouples from the observable sector, and is unimportant for particle physics phenomenology.

Recently, however, there has been a resurgence of interest [3] 12] in the phenomenology of a class of models where usual gauge interactions, not gravity, serve as the messengers of SUSY breaking. Here, it is assumed that there is a new set of particles that directly couple to the SUSY breaking sector, and which also have non-vanishing SM interactions. The idea then is that these new particles, which first feel the effects of SUSY breaking, convey these down to the observable sector via their gauge interactions. This framework differs from the mSUGRA framework in two important ways. First, because SUSY breaking is fed down to the observable sector by gauge interactions, the mass difference between ordinary particles and their superpartners depends on their gauge interactions - gluinos are thus heavier than $S U(2)$ gauginos which are heavier than binos, and $m_{\tilde{q}}>m_{\tilde{\ell}_{L}} \sim m_{\tilde{\nu}}>m_{\tilde{\ell}_{R}}$. Second, since gravitational interactions are not the messengers of SUSY breaking, $M_{o b s}$ is not suppressed by $M_{\text {Planck }}$ as in mSUGRA. Instead, we now have sparticle masses $\sim \alpha\left\langle F_{M}\right\rangle / M$, where $\mathrm{M}$ is the mass scale of the messenger particles and $\left\langle F_{M}\right\rangle$ the induced SUSY breaking scale in the messenger sector, with $\alpha$ the relevant gauge coupling of the sparticle. The important thing is that it is possible to get weak scale sparticle masses with $\left\langle F_{M}\right\rangle \sim 100 \mathrm{TeV}$ if $M$ is 100$1000 \mathrm{TeV}$. Indeed it could well be that the intrinsic SUSY breaking scale $\langle F\rangle$ is of the same magnitude, and supersymmetry breaking is determined by $100 \mathrm{TeV}$ scale physics. If this is the case, then the gravitino mass $m_{\tilde{G}}=\langle F\rangle /\left(\sqrt{3} M_{P}\right)$ (here $M_{P}=M_{\text {Planck }} / \sqrt{8 \pi}$ ) could be $\mathcal{O}(1 \mathrm{eV})$. This is important because (the longitudinal components of) such superlight gravitinos have phenomenologically relevant couplings to ordinary particles [13.

In the specific model that we adopt for our analysis, the messenger sector comprises of $n_{5}$ sets of "quark" and "lepton" superfields in a $5+\overline{5}$ representation of $S U(5)$, with superpotential couplings to a gauge singlet superfield $(\hat{S})$ which acquires vacuum expectation values for both its scalar $(S)$ and auxiliary $\left(F_{M}\right)$ components. The former results in masses for messenger quark and lepton superfields, whereas the latter is the result of the feed down of SUSY breaking from the hidden sector. Assuming that $\left\langle F_{M}\right\rangle \ll\langle S\rangle^{2}$, the gauginos acquire a mass

$$
m_{\tilde{\lambda}_{i}}=\frac{\alpha_{i}}{4 \pi} n_{5} \Lambda,
$$

whereas scalar components of chiral superfields get a mass,

$$
m_{\text {scalar }}^{2}=2 n_{5} \Lambda^{2}\left[C_{3}\left(\frac{\alpha_{3}}{4 \pi}\right)^{2}+C_{2}\left(\frac{\alpha_{2}}{4 \pi}\right)^{2}+\frac{3}{5}\left(\frac{Y}{2}\right)^{2}\left(\frac{\alpha_{1}}{4 \pi}\right)^{2}\right],
$$

with $\Lambda=\left\langle F_{M}\right\rangle /\langle S\rangle$, and $\alpha_{1}$ given in terms of the usual hypercharge coupling $g^{\prime}$ by $\alpha_{1}=\frac{5}{3} \frac{g^{\prime 2}}{4 \pi}$. Finally, $C_{3}=\frac{4}{3}$ for colour triplets and zero for colour singlets while $C_{2}=\frac{3}{4}$ for weak doublets 
and zero for weak singlets. These relations, which are independent of the messenger sector superpotential couplings $\lambda_{i}$ get corrections of $\sim\left\langle F_{M}\right\rangle /\left(\lambda_{i}\left\langle S^{2}\right\rangle\right)$ which are ignored in the subsequent analysis. The gravitino mass is given by,

$$
m_{\tilde{G}}=\frac{\langle F\rangle}{\sqrt{3} M_{P}}=\frac{\langle F\rangle}{\lambda\left\langle F_{M}\right\rangle} \frac{M \Lambda}{\sqrt{3} M_{P}} \equiv C_{G r a v} \frac{M \Lambda}{\sqrt{3} M_{P}} .
$$

The parameter $C_{\text {Grav }}$ that we have introduced is $\geq 1$ since the SUSY breaking scale in the messenger sector cannot be larger than the intrinsic SUSY breaking scale in the hidden sector, and the superpotential coupling $\lambda \leq 1$. SUSY breaking $A$-parameters and the $B$ parameter are induced only at two loops so that it is reasonable to suppose that these are small. The supersymmetric $\mu$ parameter is not determined by how SUSY breaking is mediated but is fixed (up to a sign) by radiative symmetry breaking as in the mSUGRA framework.1 A complete theory that includes the dynamics of SUSY breaking will presumably yield a value of $\mu$ consistent with this. Eq. (1) and Eq. (2) should be regarded as boundary conditions for the gaugino and scalar masses valid at the messenger scale $M=\lambda\langle S\rangle$, so that these parameters need to be evolved down to the weak scale relevant for phenomenological analysis. Our framework is thus completely parametrized by

$$
\left(\Lambda, M, n_{5}, \tan \beta, \operatorname{sgn} \mu, C_{\text {Grav }}\right)
$$

which together with Standard Model parameters completely determine the masses and couplings of all particles in the observable sector. The messenger mass scale $M$ can range from $\Lambda$ to $M_{G U T}$ or $M_{P}$. We focus our attention on models where $M \sim 1 \mathrm{PeV}$, so that the SUSY breaking scale $\left\langle F_{M}\right\rangle$ (and possibly $\langle F\rangle$ ) may be relatively low.

In order to facilitate simulation of models with gauge mediated supersymmetry breaking (GMSB) we have recently included in ISAJET v7.37 [14] a 'GMSB option' that allows one to use the GMSB parameter set introduced above as an input. ISAJET then evolves the gaugino and scalar masses as given by Eq. (11) and Eq. (2) from the scale $M$ down to the scale relevant for phenomenology, and computes the "MSSM parameters" that are then used in the evaluation of sparticle cross sections and decay patterns. The decays of neutralinos into gravitinos, $\widetilde{Z}_{i} \rightarrow \tilde{G} \gamma, \widetilde{Z}_{i} \rightarrow \tilde{G} Z$ and $\widetilde{Z}_{i} \rightarrow \tilde{G} h, H, A$ as well as (approximately) the Dalitz decay $\widetilde{Z}_{i} \rightarrow e^{+} e^{-} \tilde{G}$ are included in ISAJET. The decays $\tilde{\ell}_{R} \rightarrow \ell \tilde{G}$ and $\tilde{\tau}_{1} \rightarrow \tau \tilde{G}$, as well as the three body decays 15 (mediated by a virtual neutralino) $\tilde{\ell}_{R} \rightarrow \tilde{\tau}_{1} \bar{\tau} \ell$ and $\tilde{\ell}_{R} \rightarrow \bar{\tau}_{1} \tau \ell$ have also been included. Widths for corresponding three body decays mediated by virtual chargino exchange are suppressed by the lepton Yukawa coupling, and have not yet been included.

\footnotetext{
${ }^{1}$ It is customary to eliminate $\tan \beta$ in favour of $B_{0}$, the $B$ parameter at the scale $M$. Since $B_{0}$ is small in this framework, this constrains $\tan \beta$ to be $20-30$, the precise value depending on the choice of $\Lambda$. As in Ref. [7], we do not impose this constraint since we expect that it will be altered by new interactions introduced to generate $\mu$ dynamically.

${ }^{2}$ It is possible to construct [4] more complicated models which lead to different boundary conditions for scalar and gaugino masses from Eq. (1) and Eq. (2). We do not consider such scenarios in our analysis.
} 
These can be significant only when $m_{\tilde{\mu}_{R}}-m_{\tilde{\tau}_{1}} \lesssim m_{\tau}$ so that the neutralino-mediated three body decays of $\tilde{\mu}_{R}$ are kinematically very suppressed or forbidden.

Within the GMSB framework, the parameter $\Lambda$ sets the scale of sparticle masses. The dependence on the messenger scale $(M)$, which specifies where the boundary conditions (1) and (2) hold is presumably logarthmic. To help the reader link the model parameters with the more familiar sparticle masses, we show in Fig. 1 contours of $m_{\tilde{g}}$ in the $\Lambda-\tan \beta$ plane for $(a) n_{5}=1$, and $(b) n_{5}=2$. We fix $m_{t}=175 \mathrm{GeV}, M=1 \mathrm{PeV}$ and take $\mu>0$. We have checked that the spectrum is only weakly sensitive to $M$ for a range of $M$ between $\Lambda$ and $1 \mathrm{PeV}$, and also that it is insensitive to the sign of $\mu$ except for very low values of $\tan \beta$. The $S U(2)$ and $U(1)$ gaugino masses are roughly given by $\frac{1}{3} m_{\tilde{g}}$ and $\frac{1}{6} m_{\tilde{g}}$, respectively. The scales of the sfermion masses can roughly be read off from Eq. (2). In Region I below the diagonal solid line 3 in frame $(a), m_{\widetilde{Z}_{1}}<m_{\tilde{\tau}_{1}}$, so that $\widetilde{Z}_{1} \rightarrow \tilde{G} \gamma$ (this dominates the decays to $Z$ or Higgs bosons), so that isolated photons are a hallmark of SUSY within this framework. In Region II, sparticles cascade decay to $\tilde{\tau}_{1}$ (except immediately above this line where $m_{\widetilde{Z}_{1}}-m_{\tilde{\tau}_{1}}<m_{\tau}$ ), which then decays to $\tau \tilde{G}$, so that for this portion of the parameter space SUSY events contain $\tau$ leptons in addition to other leptons and jets [9]. In frame (b), $\widetilde{Z}_{1}$ is once again the next to lightest SUSY particle (NLSP) in Region I, while in the other regions $\tilde{\tau}_{1}$ is the NLSP (though $m_{\tilde{e}_{R}}$ and $m_{\tilde{\mu}_{R}}$ may be very close to $m_{\tilde{\tau}_{1}}$ ). In Region II, $m_{\tilde{\ell}_{R}}>m_{\widetilde{Z}_{1}}>m_{\tilde{\tau}_{1}}$, while in Regions III and IV, $\widetilde{Z}_{1} \rightarrow \tilde{\ell}_{R} \ell$ is kinematically possible. In Region III, $\tilde{\ell}_{R} \rightarrow \ell \tilde{G}$ because the decay $\tilde{\ell}_{R} \rightarrow \tau \tilde{\tau}_{1} \ell$ is kinematically inaccessible, while in Region IV, both these decays are possible and compete. The phenomenology will clearly be sensitive to just where we are in the parameter space.

The grey region on top of each frame is excluded because proper electroweak symmetry breaking is not obtained: the wedge in the upper left corner is excluded because $m_{\tilde{\tau}_{R}}^{2}<0$ while in the band on the top, $m_{A}^{2}<0$. The various hatched regions are excluded by the non-observation of sparticles or a Higgs boson at LEP 2. In the region with vertical shading, $m_{\tilde{\tau}_{1}}<60 \mathrm{GeV}$ (in the corresponding portion of Region I, this limit may be stronger as the final state also contains photons); in that shaded horizontally, $m_{\widetilde{W}_{1}}<88 \mathrm{GeV}$; in the small diagonally shaded region at low $\tan \beta, m_{h}<89 \mathrm{GeV}$ (the current lower limit on the SM Higgs boson mass). While these limits [17] are essentially those obtained in the mSUGRA model, the strongest constraint comes from the non-observation of acollinear photon pairs from $e^{+} e^{-} \rightarrow \widetilde{Z}_{1} \widetilde{Z}_{1} \rightarrow \gamma \gamma \tilde{G} \tilde{G}$, and is special to this framework. The ALEPH collaboration 18] has excluded $m_{\widetilde{Z}_{1}}<71 \mathrm{GeV}$ assuming that $\widetilde{Z}_{1} \approx \tilde{B}$ and $m_{\tilde{e}_{R}}=1.5 m_{\widetilde{Z}_{1}}$, which is a good approximation for $n_{5}=1$. This exclusion is shown as the diagonally shaded vertical strip in

\footnotetext{
${ }^{3}$ There are finite one loop corrections [16] which alter the relationship between the fermion mass and the corresponding Yukawa coupling that have not been included in ISAJET. These corrections, which can be important for large $\tan \beta$, would shift the exact location of this line. Thus, for model parameter values close to this line, these corrections could radically alter the phenomenology if the NLSP shifts from being a neutralino to being a stau. While these corrections are crucial to include in any study relating SUSY phenomena to the underlying parameters (that may be determined by physics at yet higher energy scales) of the theory, this issue is not critical to our main point which is a study of the LHC reach for the case where $\widetilde{Z}_{1}$ is the NLSP.
} 
the figures. T The D0 experiment at the Tevatron [19], from a non-observation of di-photon events with large $E_{T}$ have inferred a lower bound $\sim m_{\widetilde{W}_{1}}>150 \mathrm{GeV}$, which essentially excludes this same region.

We have already noted that if model parameters happen to be in Region I of Fig. 1, in addition to multijet and multilepton events expected in the mSUGRA framework, SUSY events would contain, in addition, hard isolated photons, provided that the SUSY breaking scale $\langle F\rangle$ is not much larger than $\left\langle F_{M}\right\rangle$ for the messenger sector, i.e., that $C_{\text {grav }}$ is not too large. In Region II (and III and IV in frame $b$ ), SUSY events would contain additional $\tau$ (and possibly $e$ or $\mu$ ) instead of photons. The main purpose of this study is to assess the SUSY reach of the Large Hadron Collider within the GMSB framework, assuming that the parameters are in Region I. For this reason, we will mainly focus on the case $n_{5}=1$ in the rest of the analysis and defer the study of the phenomenology where sleptons are lighter than $\widetilde{Z}_{1}$ to a subsequent analysis.

We begin by showing the production cross sections as a function of the parameter $\Lambda$ for a variety of sparticle production reactions in Fig. 2 . We have fixed $M=1 \mathrm{PeV}, n_{5}=1$, $\mu>0$ which we take to be our default values, and taken $\tan \beta=2$. The cross sections are independent of $C_{\text {Grav }}$. We use CTEQ3L structure functions [20] for our analysis. We see that squark and gluino production dominates for small values of $\Lambda \lesssim 175 \mathrm{TeV}$, beyond which electroweak production of (lighter) charginos and neutralinos is the dominant source of sparticles. The production of charginos or neutralinos in association with gluinos or squarks is always sub-dominant. The dotted curve, which includes cross sections for $\tilde{\ell} \tilde{\ell}, \tilde{\ell} \tilde{\nu}$ and $\tilde{\nu} \tilde{\nu}$ production, shows that slepton pair production is an order of magnitude smaller than chargino-neutralino production. Except when $\Lambda \simeq 50-100 \mathrm{TeV}$ where $\tilde{\nu} \tilde{\ell}_{L}$ production is also important, slepton production is dominated by $\tilde{\ell}_{R} \tilde{\ell}_{R}$ production $(\tilde{\ell}=e, \mu$ in the figure), a reflection of the fact that $2 m_{\tilde{\ell}_{R}} \simeq m_{\tilde{\ell}_{L}} \sim m_{\tilde{\nu}}$. It is interesting to observe that $\tilde{\ell}_{R} \tilde{\ell}_{R}$ production which results in the essentially SM background-free $\ell \ell \gamma \gamma+E_{T}$ events may be observable (if they can be separated from other SUSY sources of dileptons) for $\Lambda$ values as large as $225 \mathrm{TeV}$, corresponding to $m_{\tilde{\ell}_{R}}=400 \mathrm{GeV}$.

For detector simulation at the LHC, we use the toy calorimeter simulation package ISAPLT. We simulate calorimetry covering $-5<\eta<5$ with cell size $\Delta \eta \times \Delta \phi=0.05 \times 0.05$. We take the hadronic energy resolution to be $50 \% / \sqrt{E} \oplus 0.03$ for $|\eta|<3$, where $\oplus$ denotes addition in quadrature, and to be $100 \% / \sqrt{E} \oplus 0.07$ for $3<|\eta|<5$, to model the effective $p_{T}$ resolution of the forward calorimeter including the effects of shower spreading, which is otherwise neglected. We take electromagnetic resolution to be $10 \% / \sqrt{E} \oplus 0.01$. Although we have included these resolutions, which are typical of ATLAS [21] and CMS [22], we have made no attempt to estimate the effects of cracks, edges, and other problem regions. Much more detailed detector simulations are needed to understand the effects of such regions and of the resulting non-Gaussian tails, particularly on the $\mathbb{E}_{T}$ resolution.

Jets are found using fixed cones of size $R=\sqrt{\Delta \eta^{2}+\Delta \phi^{2}}=0.7$ using the ISAJET routine GETJET. Clusters with $E_{T}>100 \mathrm{GeV}$ and $\mid \eta($ jet $) \mid<3$ are labeled as jets. Muons and

\footnotetext{
${ }^{4}$ For $n_{5}=2$ in Fig. $1 b$, although we have shown the same limit of $71 \mathrm{GeV}$, the reader should be aware that this is overly conservative because, for any given value of $m_{\widetilde{Z}_{1}}, m_{\tilde{e}_{R}}$ will be lighter than for $n_{5}=1$, and the $\widetilde{Z}_{1} \widetilde{Z}_{1}$ cross section will be correspondingly larger.
} 
electrons are classified as isolated if they have $p_{T}>10 \mathrm{GeV},|\eta(\ell)|<2.5$, and the visible activity within a cone of $R=0.3$ about the lepton direction is less than $E_{T}$ (cone) $=5 \mathrm{GeV}$. Photons with $E_{T}>10 \mathrm{GeV}$ and $|\eta|<2.5$ are considered to be isolated if there is less than $2.5 \mathrm{GeV}$ of other activity in a cone of $R=0.3$ about the photon.

To enhance the SUSY signal over SM background processes, we require that each event contains at least two entities (an entity is a jet, an electron, a muon or a photon) with $E_{T}>100 \mathrm{GeV}$ and $E_{T}>100 \mathrm{GeV}$ and at least one isolated photon with $E_{T}>40 \mathrm{GeV}$. The inclusive two photon sample is required to have a second isolated photon with $E_{T}>25 \mathrm{GeV}$. As in previous analyses [23,24], we find it useful to use variable cuts for the two hardest entities and $E_{T}$ : we require $E_{T 1}, E_{T 2}, E_{T} \geq E_{T}^{c}$, where we vary the parameter $E_{T}^{c}$.

To estimate SM background we have not done a new simulation, but used our previous computations of backgrounds for SUSY signals without photons to obtain an estimate of the background level for the present situation. The background cross sections as obtained from Fig. 3 of Ref. [24] are shown in the first three rows of Table 1 for several values of $E_{T}^{c}$. In this computation, the basic lepton and jet identification requirements were identical to the ones used in the present analysis and $E_{T}(j 1), E_{T}(j 2)$ and $E_{T}$ were all required to be larger than $E_{T}^{c}$. Radiation of each hard isolated (i.e. large angle) photon is suppressed by a factor $\alpha$ (we neglect coefficients of $\mathcal{O}(1)$ and factors of $2 \pi$ ), so that we may estimate the physics backgrounds for SUSY events with one or two isolated photons by multiplying the total background in Ref. [24] by $\alpha$ and $\alpha^{2}$, respectively. This background level is shown in the next two rows of the Table. What about non-physics backgrounds from mis-identification of jets or leptons as photons? We estimate this by assuming that the jet gamma rejection is $1 / 5000$ [25], and that SUSY events typically have a jet multiplicity $\sim 10$. The chance of an electron to fake a photon is much smaller $\left(\sim 10^{-5}\right)$ so that this background is negligible. We may thus obtain a crude estimate of the non-physics backgrounds to single (two) photon events by reducing the total background in Ref. [24 by a factor of $500\left(\frac{45}{5000^{2}} \sim 2 \times 10^{-6}\right)$. This is shown in the last two rows of Table 1. An important conclusion that one draws from the Table is that while the detector-dependent background to single photon events may be comparable to the physics background, it is relatively unimportant for the inclusive two photon SUSY event sample.

The reader may worry that the background sample always contains two jets with $E_{T}>$ $E_{T}^{c}$, while a portion of the signal may instead contain leptons or photons instead of jets as the primary entities, the background to which has not been included in Ref. [24]. While we have not estimated this, we do not expect it to be a problem since it is reasonable to suppose that in the SM, events with very hard isolated leptons and/or photons are much rarer than those with hard jets.

In order to estimate the SUSY reach of the LHC, we have computed the signal cross sections for the one and two-photon inclusive SUSY samples. Typically, we found that after acceptance cuts, the two photon inclusive signal rate is suppressed relative to the corresponding single photon rate by a factor of $2-3$, depending on sparticle masses. Since two photon backgrounds are much more strongly suppressed the best reach is obtained in the $\gamma \gamma+E_{T}+X$ channel. We show LHC cross sections for such events versus $\Lambda$ after cuts in Fig. 3 for $(a) \mu>0, \tan \beta=2,(b) \mu>0, \tan \beta=35$, and $(c) \mu<0, \tan \beta=2$. The parameters $n_{5}$ and $M$ are set at our default values. In our computation, we assume that the decay $\widetilde{Z}_{1} \rightarrow \gamma \tilde{G}$ occurs instantaneously; i.e. the photon emerges from the primary 
vertex. (The typical decay length is millimeters for $C_{\text {grav }}=1$. This is short enough to be unimportant for photons but is easily measured via $\widetilde{Z}_{1} \rightarrow e^{+} e^{-} \tilde{G}$ Dalitz decays.) Also shown is the background level, estimated as described above. We show cross sections for $E_{T}^{c}=100 \mathrm{GeV}$ (solid) and $200 \mathrm{GeV}$ (dashed). The scale on the top axis is the gluino mass, which is essentially the same for each of the frames. We see that by choosing the larger value of $E_{T}^{c}$, especially for $\Lambda \gtrsim 200-250 \mathrm{GeV}$, the SM background can be greatly reduced with only modest loss of signal. Indeed, the signal is then only rate limited, and we obtain a 5 event level, which we take to define the reach of the LHC, with an integrated luminosity of $10 \mathrm{fb}^{-1}$ if $\Lambda \lesssim 400 \mathrm{TeV}$ (corresponding to $m_{\tilde{g}} \lesssim 2.8 \mathrm{TeV}$ ) in all three frames. The expected SM background is 0.5 event, so that the Poisson probablility of fluctuation to give more than five events is $1.4 \times 10^{-5}$. Of course, one must view this number in perspective, considering the crudeness of our background estimate. Note however, that even if the background is larger than our estimate by an order of magnitude, the typical experiment would exclude the SM at $99 \%$ CL if $\Lambda \lesssim 380 \mathrm{TeV}$, or a " $5 \sigma$ discovery" would be possible for $\Lambda \lesssim 300 \mathrm{TeV}$.

We remark that we have also checked for several cases along the boundary of Region I in Fig. $1 b$ that the SUSY signal is observable even if $n_{5}=2$, as long as $\widetilde{Z}_{1} \rightarrow \tilde{G} \gamma$ well inside the detector. We expect that the signal is observable over this entire region.

Finally, we briefly consider prospects for determining the parameter $\Lambda$ that sets the sparticle mass scale in our scenario. At first, it might seem that the mean values of the total scalar energy, the visible invariant mass, or the $E_{T}$ of the hardest entities in SUSY events would scale with $\Lambda$. This is, however, not the case because the dominant SUSY sources change from $\tilde{g}$ and $\tilde{q}$ to (much lighter) charginos and neutralinos as $\Lambda$ increases from below $200 \mathrm{TeV}$ to above $200 \mathrm{TeV}$. The mass of $\widetilde{Z}_{1}$ (which is dominantly a bino), on the other hand always scales with $\Lambda$, so that it is reasonable to expect that the $p_{T}$ of the photons produced via its decays to the gravitinos would scale similarly. To illustrate this we show in Fig. 4 the $p_{T}$ distribution of the hard photon in SUSY events where both photons are detected for several values of $\Lambda$. For definiteness, we have illustrated this for four values of $\Lambda$ with other parameters as in Fig. $3 a$, and for $E_{T}^{c}=100 \mathrm{GeV}$. Assuming that our background estimate is valid, we see that the distributions show a clear scaling with $\Lambda$, though for the highest value of $\Lambda$ an integrated luminosity of $30-50 \mathrm{fb}^{-1}$ would be required to construct this distribution. The $p_{T}$ distribution of the second photon shows the same scaling behaviour.

Although the $p_{T}(\gamma)$ distributions in Fig. 4 scale nicely with $\Lambda$, it is not enough to determine $\Lambda$ as $n_{5}$ is not known. The most obvious strategy for the determination of $n_{5}$ is to obtain the ratio of sfermion and gaugino masses. This may, for example, be possible by focussing on event chains [26] in which the decays $\widetilde{Z}_{2} \rightarrow \ell \tilde{\ell}_{R} \rightarrow \ell \bar{\ell} \widetilde{Z}_{1} \rightarrow \ell \bar{\ell} \gamma \tilde{G}$ (for $n_{5}=1$ ) or even $\widetilde{Z}_{1} \rightarrow \tilde{\ell}_{R} \ell \rightarrow \ell \bar{\ell} \tilde{G}$ (for $n_{5}=2$ ) can be isolated. Additional information may also be available from the overall mass and $E_{T}$ of the hardest entities in SUSY events. The distribution of decay lengths of the NLSP, which may be possible to determine from the displacement of the secondary vertex can yield information about the underlying SUSY breaking scale. A study of these issues, and also the reach of the LHC when the NLSP is not the lightest neutralino, is in progress.

Acknowledgement: We are grateful to Uri Sarid and Carlos Wagner for bringing the importance of the corrections in Ref. [16 to our attention. P.M. was partially supported by Fundação de Amparo à Pesquisa do Estado de São Paulo (FAPESP). HB thanks the Davis Institute for High Energy Physics for hospitality while a portion of this work was 
completed. This research was supported in part by U.S. Department of Energy Grants DE-FG-03-94ER40833, DE-FG-02-97ER41022 and DE-AC02-76CH00016. 


\section{TABLES}

TABLE I. SM background cross sections from Fig. 3 in Ref. 24] as a function of $E_{T}^{c}$ defined in the text. The $2 \ell$ background includes contributions from both same sign and opposite sign leptons. Trilepton background cross sections are much smaller. The next two rows, which are obtained by multiplying the total background by $\alpha \sim 10^{-2}$ and $\alpha^{2} \sim 10^{-4}$, give our estimate of physics sources for single photon and two photon inclusive events passing the SUSY selection cuts. Detector-dependent backgrounds, which are estimated as discussed in the text, are shown in the last two rows.

\begin{tabular}{|l|c|c|c|}
\hline \hline$E_{T}^{c}(\mathrm{GeV})$ & 100 & 200 & 300 \\
\hline$\sigma\left(E_{T}\right)(f b)$ & $10^{4}$ & 500 & 50 \\
$\sigma(1 \ell)(f b)$ & 1200 & 30 & 4 \\
$\sigma(2 \ell)(f b)$ & 700 & 10 & 0.4 \\
\hline$\alpha \times \sigma_{T o t}(f b)$ & 100 & 5 & 0.5 \\
$\alpha^{2} \times \sigma_{T o t}(f b)$ & 1 & 0.05 & 0.005 \\
\hline Fake $(1 \gamma)(f b)$ & 20 & 1 & 0.1 \\
Fake $(2 \gamma)(f b)$ & 0.02 & $10^{-3}$ & $10^{-4}$ \\
\hline \hline
\end{tabular}




\section{REFERENCES}

[1] For recent reviews of the phenomenology of SUSY, see H. Baer et. al., in Electroweak Symmetry Breaking and New Physics at the TeV Scale, edited by T. Barklow, S. Dawson, H . Haber and J. Seigrist, (World Scientific, 1995); M. Drees, hep-ph/9611409 (1997); X. Tata, Lectures presented at the IX Jorge Swieca Summer School, Campos do Jordão, Brazil, Feb. 1997, UH-511-872-97, hep-ph/9706307 (1997); S. Dawson, Lectures at TASI 97, hep-ph/9712464 (1997); M. Carena et al. hep-ex/9712022 (1997); F. Paige, Lectures at TASI 97, hep-ph/9801254 (1998).

[2] For a review see, H. P. Nilles, Phys. Rep. 110, 1 (1984).

[3] M. Dine and A. Nelson, Phys. Rev. D48, 1277 (1993); M. Dine, A. Nelson, Y. Shirman, Phys. Rev. D51, 1362 (1995); M. Dine, A. Nelson, Y. Nir and Y. Shirman, Phys. Rev. D53, 2658 (1996).

[4] S. Dimopoulos, M. Dine, S. Raby and S. Thomas, Phys. Rev. Lett. 76, 3494 (1996); S. Dimopoulos, S. Thomas and J. Wells, Phys. Rev. D54, 3283 (1996) and Nucl. Phys. B488, 39 (1997)

[5] S. Ambrosanio, G. Kane, G. Kribs, S. Martin and S. Mrenna, Phys. Rev. Lett. 76, 3498 (1996) and Phys. Rev. D54, 5395 (1996).

[6] K. S. Babu, C. Kolda and F. Wilczek, Phys. Rev. Lett. 77, 3070 (1996).

[7] H. Baer, M. Brhlik, C. H. Chen and X. Tata, Phys. Rev. D55, 4463 (1997).

[8] J. Bagger, K. Matchev and D. Pierce, Phys. Rev. D55, 3188 (1997).

[9] D. Dicus, B. Dutta and S. Nandi, Phys. Rev. Lett. 78, 3055 (1997) and Phys. Rev. D56, 5748 (1997); K. Cheung, D. Dicus, B. Dutta and S. Nandi, hep-ph/9711216 (1997).

[10] C. H. Chen and J. F. Gunion, Phys. Lett. B420, 77 (1998) and hep-ph/9802252 (1998).

[11] J. Feng and T. Moroi, hep-ph/9712499 (1997).

[12] For a review, see G. Giudice and R. Rattazzi, hep-ph/9801271 (1998).

[13] P. Fayet, Phys. Lett. B70, 461 (1977).

[14] ISAJET 7.37, F. Paige, S. Protopopescu, H. Baer and X. Tata, hep-ph/9804321 (1998).

[15] S. Ambrosanio, G. Kribs and S. Martin, Nucl.Phys. B516, 55 (1998).

[16] R. Hempfling, Z. Phys. C63, 309 (1994); R. Hempfling and B. Kniehl, Phys. Rev. D51, 1386 (1995); L. Hall, R. Rattazzi and U. Sarid, Phys. Rev. D50, 7048 (1994); M. Carena, M. Olechowski, S. Pokorski and C. Wagner, Nucl. Phys. B426, 269 (1994).

[17] See e.g. Talk presented by J. Mnich at PASCOS-98, Northeastern University, March 1998.

[18] R. Barate et al., Phys. Lett. B420, 127 (1998).

[19] B. Abbott et al. Phys. Rev. Lett. 80, 442 (1998); see also S. Abachi et al. Phys. Rev. Lett. 78, 2070 (1997) and F. Abe et al. hep-ex/9801019 (1998).

[20] H. L. Lai et al., Phys. Rev. D51, 4763 (1995).

[21] ATLAS Technical Proposal, CERN/LHCC/94-43 (1994).

[22] CMS Technical Proposal, CERN/LHCC 94-38 (1994).

[23] H. Baer, C. H. Chen, F. Paige and X. Tata, Phys. Rev. D52, 2746 (1995).

[24] H. Baer, C. H. Chen, F. Paige and X. Tata, Phys. Rev. D53, 6241 (1996).

[25] F. Gianotti, private communication.

[26] A. Bartl et al., LHC Subgroup Report in New Directions for High Energy Physics, Proc. Snowmass 96 Workshop, D. Cassel, L. Trindle Gennari and R. Siemann, Editors; I. Hinchliffe et al. Phys. Rev. D55, 5520 (1997). 

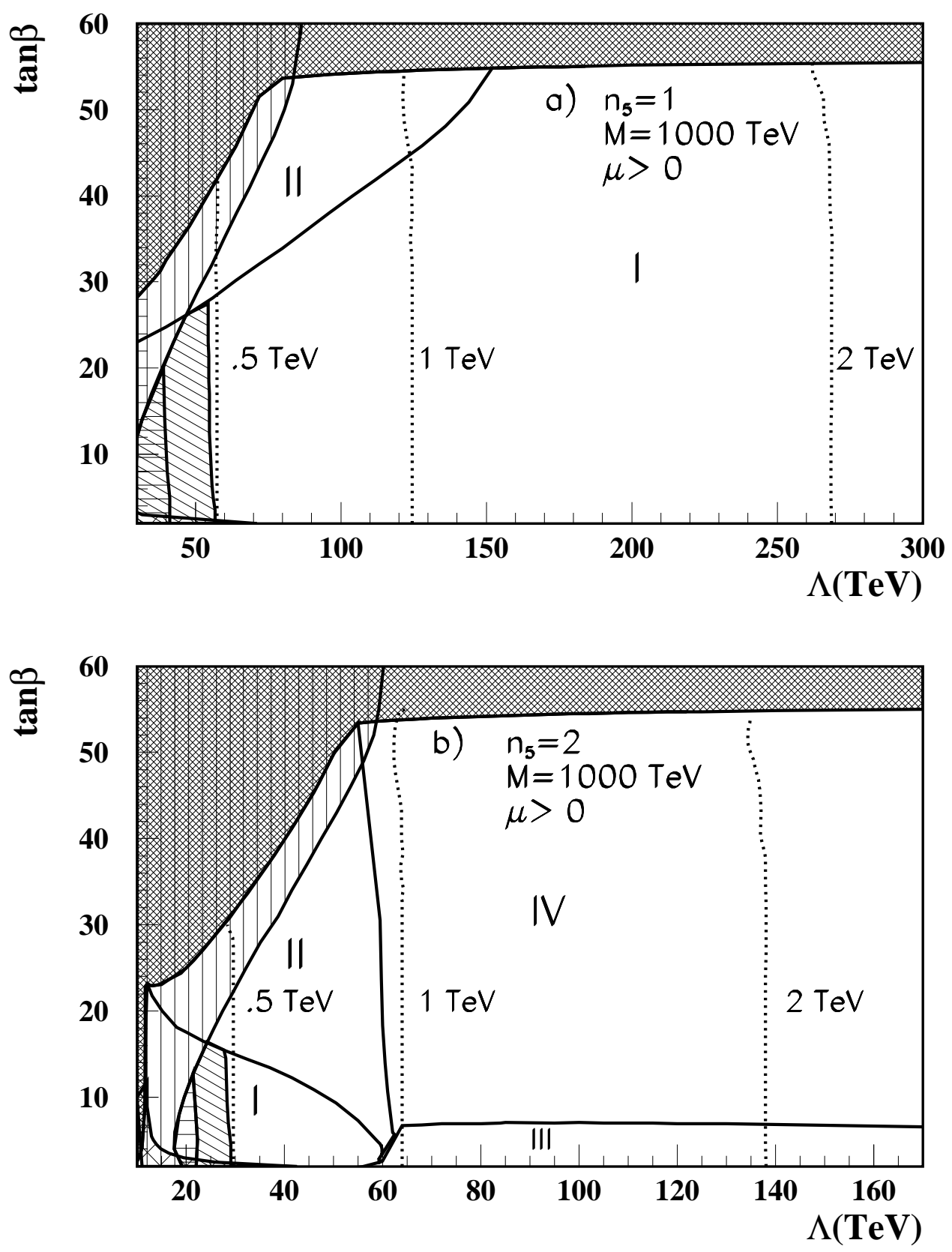

FIG. 1. Various regions in the $\Lambda-\tan \beta$ plane for $M=1000 \mathrm{TeV}$ and $\mu>0$ for $a) n_{5}=1$ and $b$ ) $n_{5}=2$. The grey region is excluded by theoretical considerations while the various hatched regions are excluded by experimental constraints discussed in the text. Also shown by the dotted curves are contours of gluino mass. In Region I, $\widetilde{Z}_{1}$ is the NLSP while in the other regions, the NLSP is a slepton. 


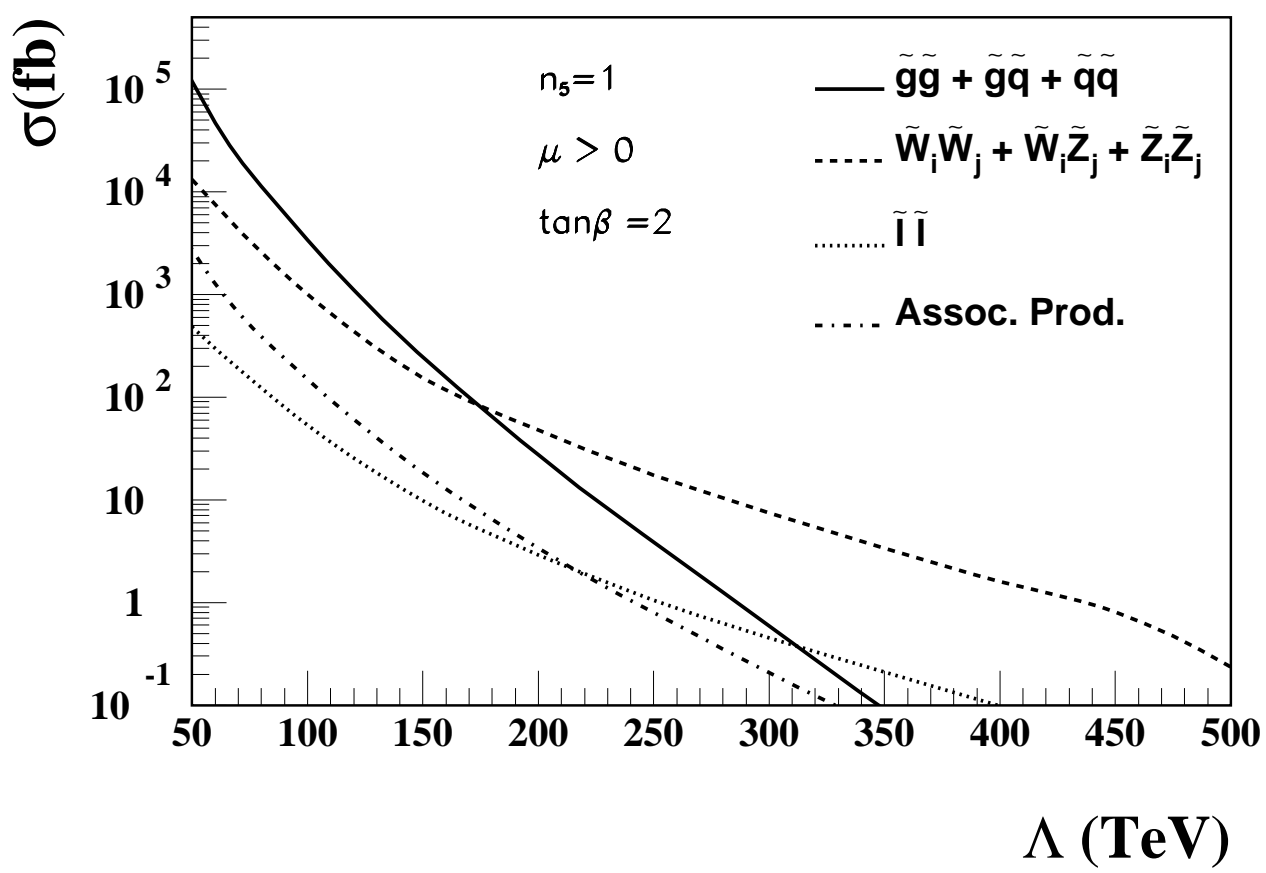

FIG. 2. The production cross sections versus $\Lambda$ for various sets of SUSY processes at a $14 \mathrm{TeV}$ $p p$ collider within the GMLESB framework. We show the cross sections for $M=1000 \mathrm{TeV}$, with other parameters as shown in the figure. For sleptons, we sum over the first two families. 


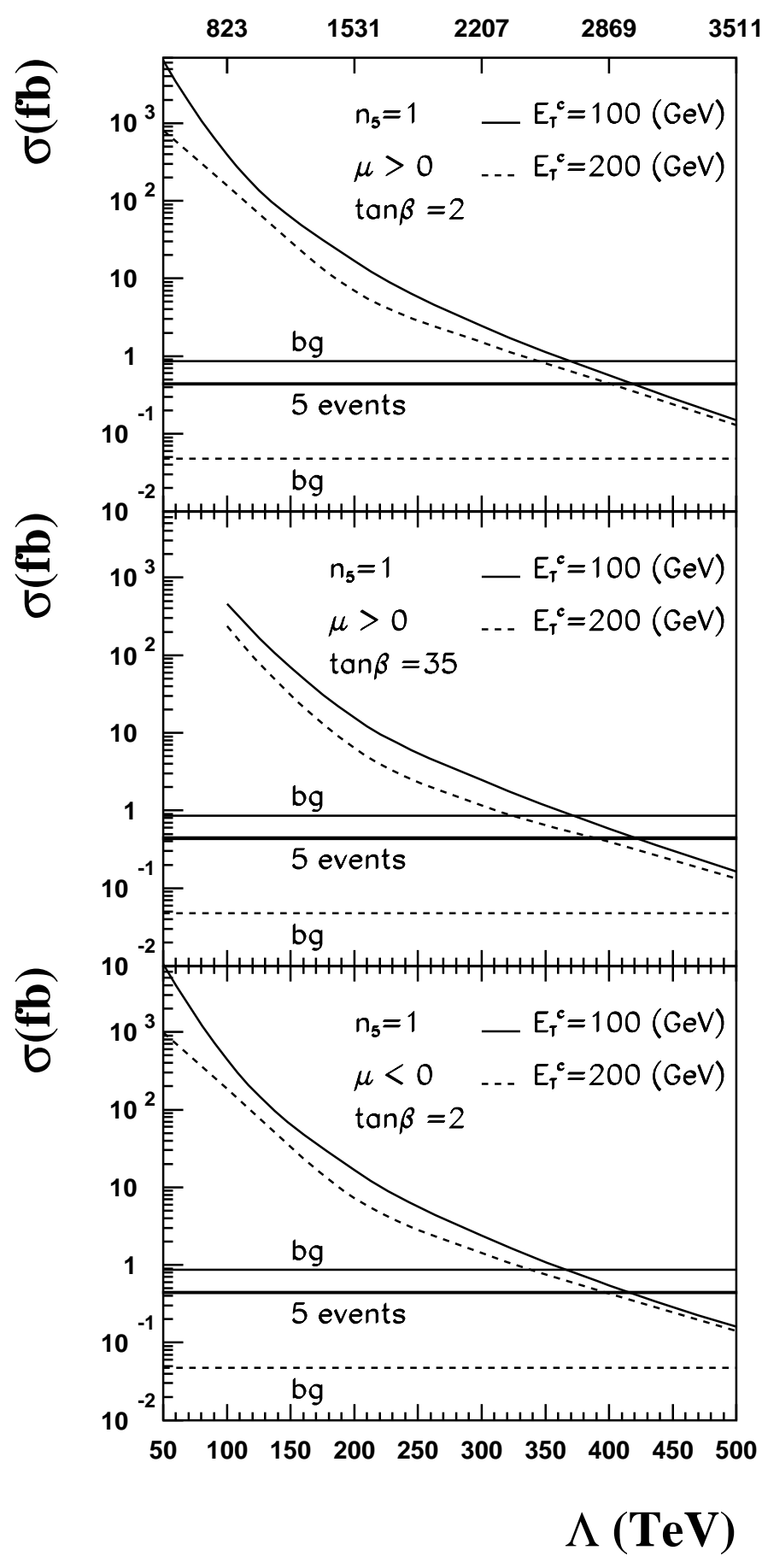

FIG. 3. Signal cross sections for inclusive $\gamma \gamma+\not_{T}+X$ events at the LHC after the cuts described in the text for two values of the cut parameter $E_{T}^{c}$. We fix $M=1000 \mathrm{TeV}$. Our estimate of the background level from SM sources is shown by the lines denoted by bg. The five event level is also shown for an integrated luminosity of $10 \mathrm{fb}^{-1}$. 


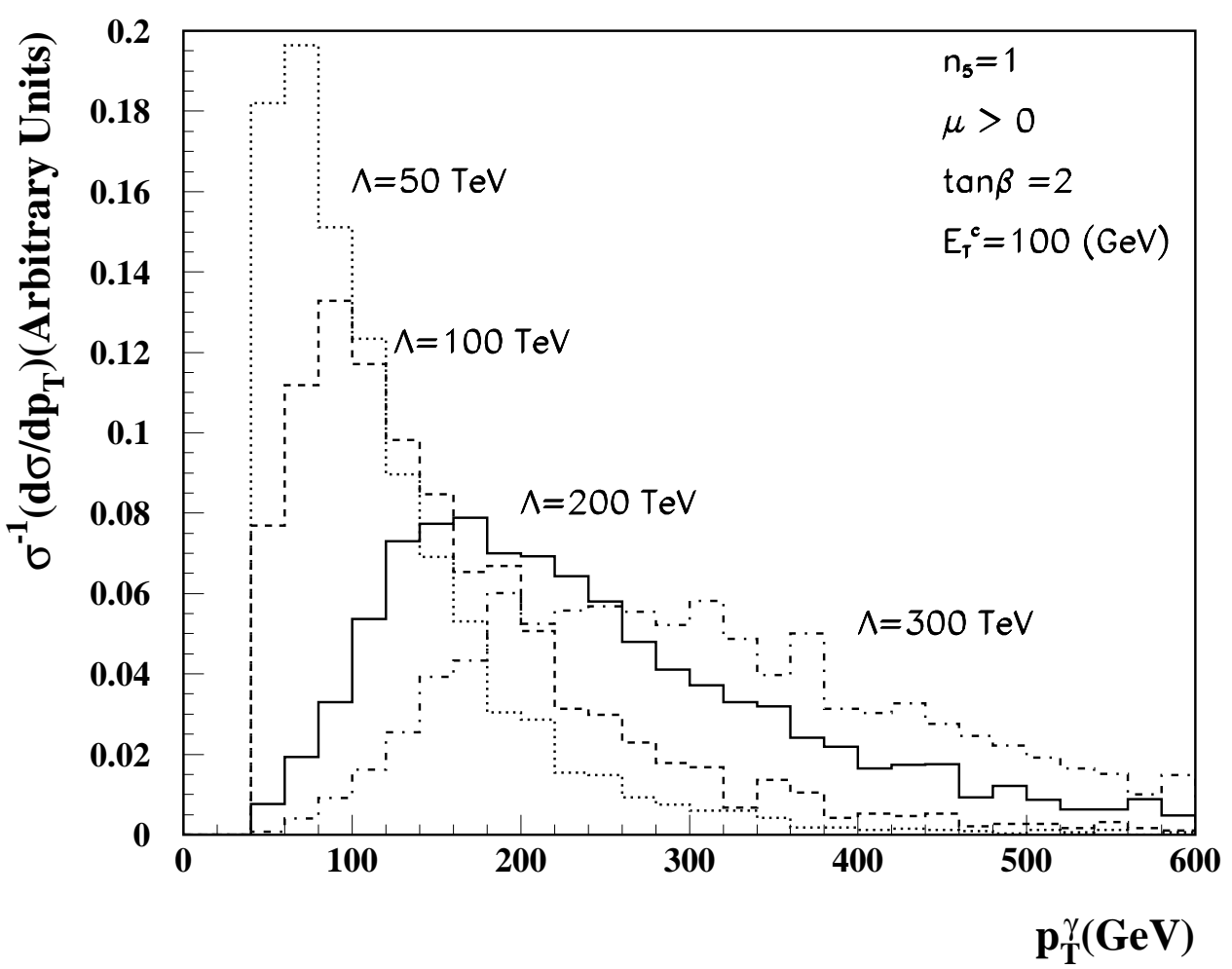

FIG. 4. The transverse momentum distributions for the hard photon in inclusive $\gamma \gamma+\not_{T}$ events at the LHC as computed within the GMSB framework for several values of $\Lambda$ and the cut parameter $E_{T}^{c}=100 \mathrm{GeV}$. We choose $M=1000 \mathrm{TeV}$. 